Ebisu Ebisu

Études japonaises Études japonaises

$57 \mid 2020$

Les architectes de l'ère Heisei (1989-2019). Rôles, statuts, pratiques et productions

\title{
STEININGER Brian, Chinese Literary Forms in Heian Japan. Poetics and Practices
}

Cambridge, Harvard University Press, 2017, 293 p.

\section{Arthur Defrance}

\section{(2) OpenEdition}

Journals

Édition électronique

URL : http://journals.openedition.org/ebisu/5435

DOI : $10.4000 /$ ebisu. 5435

ISSN : 2189-1893

Éditeur

Institut français de recherche sur le Japon à la Maison franco-japonaise (UMIFRE 19 MEAE-CNRS)

Édition imprimée

Date de publication : 15 décembre 2020

Pagination : 447-450

ISSN : 1340-3656

Référence électronique

Arthur Defrance, " steininger Brian, Chinese Literary Forms in Heian Japan. Poetics and Practices », Ebisu [En ligne], 57 | 2020, mis en ligne le 20 décembre 2020, consulté le 29 mars 2021. URL : http:// journals.openedition.org/ebisu/5435; DOI : https://doi.org/10.4000/ebisu.5435 


\section{Comptes rendus}

() Steininger Brian, Chinese Literary Forms in Heian Japan. Poetics and Practices, Cambridge, Harvard University Press, 2017, 293 p.

La richesse thématique de cet ouvrage récent de Brian Steininger peut donner le sentiment de voir l'aboutissement des nombreux travaux américains publiés sur la littérature en sinitique ${ }^{1}$ au Japon, dont la généalogie peut être retracée depuis les années $1980^{2}$, mais dont l'explosion est un phénomène plus récent, comme l'a noté John Wixted ${ }^{3}$. Ce serait néanmoins ne pas faire justice à
Steininger, qui fait bien plus que réaliser une synthèse, même si son texte semble aussi avoir été pensé comme un ouvrage pédagogique, avec ses cinq chapitres clairement découpés, ses traductions et ses séquences explicatives nombreuses et instructives. Louvrage parvient à traiter simultanément de sujets assez divers (la place de l'écriture et de la littérature, le rapport entre le sinitique et le vernaculaire japonais et l'influence qu'il a sur la dynamique des genres), qu'il lie entre eux par le truchement d'un personnage historique, Minamoto no Shitagō 源順 (911-983), et des textes qui lui sont attribués (parfois sans que l'attribution soit certaine). Ce dispositif permet de poser la question centrale du texte, celle de la place du savoir des lettrés (monnin 文人), plus 
précisément, des diplômés de la « Voie des Lettres » (monjōdō 文章道, puis $k i d e n d \bar{o}$ 紀伝道) de l'Office des études supérieures (Daigaku-ryō 大学寮) au milieu et à la fin de l'époque de Heian ( $\mathrm{x}^{\mathrm{e}}$ et $\mathrm{xI}^{\mathrm{e}}$ siècles). L'époque est précisément - et ce n'est pas un hasard - celle des textes en vernaculaire devenus canoniques, comme le Kokinshū 古今集 (Recueil de poésies anciennes et modernes), Makura no sōshi 枕草子 (Notes de chevet) et le Genji monogatari 源氏物語 (Le Dit $d u$ Genji), dans lesquels nos lettrés sont souvent caricaturés comme des besogneux, qui n'en font que mieux ressortir les aptitudes artistiques naturelles des nobles. Cette époque est ainsi celle d'une perte d'autorité pour les lettrés et Steininger tente de montrer les stratégies de maintien de légitimité que ceux-ci mettent en œuvre dans ce contexte.

Le premier chapitre s'intéresse à l'Utsubo monogatari 宇津保物語 (Le Dit de l'arbre creux), attribué - semble-t-il - à tort à Shitagō. Il s'ouvre sur une présentation assez complète de la situation de déclassement dans laquelle les lettrés se trouvent plongés (liée à une stratification croissante de la noblesse et à la confiscation du pouvoir et des charges par la maison impériale et les grandes maisons nobles). La confiscation du pouvoir a pour conséquence de faire diminuer le nombre d'occasions formelles nécessitant des compositions et des récitations en sinitique, ce qui détériore la situation économique des lettrés. Ceux-ci sont donc contraints de rechercher directement le soutien des nobles de haut rang pour obtenir de l'avancement (parfois avec l'aide des zuryō 受領, fonctionnaires chargés de la perception des taxes en province). Le lieu fantasmé de cette rencontre est le banquet privé des nobles, que l'Utsubo dépeint comme faussement égalitaire, même si certains personnages $\mathrm{du}$ récit refusent ou critiquent la logique de l'échange et du patronage.

Le deuxième chapitre se penche sur l'anthologie sinitique Honchō monzui 本朝文粋 (Fine Fleur des belles-lettres de notre nation), compilée en 1060 par Fujiwara no Akihira 藤原明衡 (989-1066). Steininger montre que l'organisation de ces 432 textes écrits entre 810 et 1037 permet de voir la position dans laquelle sont placés les lettrés, contraints de composer pour des protecteurs, qui leur demandent le plus souvent des jo 序 (préfaces poétiques), des ganmon 願文 (prières) et des hyō 表 (adresses au trône). Les lettrés ne sont pas à égalité dans cette compétition et certaines familles s'imposent au détriment d'autres, comme on le voit au fait que les auteurs repris dans l'anthologie qui reçoivent des commandes de textes utilisés pendant des rites 
officiels sont peu nombreux (Shitagō n'est pas de ce nombre). On s'étonne un peu que Steininger ne mentionne pas le texte de Sugawara no Fumitoki 菅原文時 (899-981) appelé Fūji sankajo 封事三䇢条 (Trois remarques anonymes) qui critique très directement le dédain général pour les lettres et le favoritisme à l'œuvre dans l'attribution des charges, texte qui aurait permis de souligner la nature contestataire de l'anthologie d'Akihira".

Le troisième chapitre s'intéresse plus spécifiquement au cas du parallélisme en littérature sinitique. Après une présentation très utile de la place de ce procédé en Chine et au Japon, Steininger défend l'idée que la formalisation des techniques de hadai 破題 (développement du thème en vers parallèles) a ouvert horizontalement la compétition littéraire à un plus grand nombre, non limité aux seuls lettrés professionnels. La conséquence a été une surenchère d'érudition (d'où le développement de commentaires de distiques, comme ceux du Wakan rōeish u 和漢朗詠集 [Recueil de poèmes à réciter, japonais et chinois]), le détachement de bons distiques de leur contexte, y compris politique (c'est le cas célèbre des Nouvelles Ballades [Xin-Yuefu 新楽府] de Bai Juyi 白居易), et le développement par les lettrés d'une stratégie verticale d'appel direct à un protecteur potentiel, dans des formules d'expression personnelles (jukkai 述懐) empruntant au vocabulaire de la pétition adressée au trône ( $s \overline{0} j \bar{o}$ 奏状).

Le chapitre qui suit est plus didactique, dans une certaine mesure: Steininger revient d'abord sur l'histoire de l'Office des études supérieures, des manuels utilisés pour l'apprentissage de l'écriture, puis sur celle des techniques de lecture vernaculaire (kundoku 訓読), où le rôle des textes bouddhiques est peut-être un peu trop rapidement traité (même s'il est fait référence aux analyses de John Whitman sur les manuscrits bouddhiques coréens du Japon ${ }^{5}$ ). La fin du chapitre défend l'idée que l'autorité de ce savoir académique unitaire est concurrencée par la légitimité des transmissions particulières de savoir rituel des grandes maisons, qui adoptent aussi des standards de textualité vernacularisés (ceux des journaux de cour et des lettres). Steininger conclut sur l'idée intéressante selon laquelle ces deux savoirs s'alimentent l'un l'autre, le savoir académique devenant une affaire de lignée et le savoir rituel s'appuyant de plus en plus sur l'autorité du précédent.

Le dernier chapitre est peut-être le plus ambitieux et celui qui enrichit le plus la description du kundoku de David Lurie ${ }^{6}$. Steininger reconnaît que le procédé permet de réaliser de manière similaire en japonais des 
textes écrits dans des styles sinitiques très divers (il prend l'exemple des actes de don de terrains aux temples se.nyübun 施入文, parfois très proches de l'idéal académique, parfois très vernacularisés), mais il avance l'idée qu'il y a différents niveaux dans la réalisation orale de la prose sinitique. Le dictionnaire de Shitagō, le Wamyō ruijū shō 倭名類聚抄 (Guide des mots japonais), qui distingue termes corrects (empruntés aux lectures archaïsantes du Nihon shoki 日本書紀 [Chroniques du Japon]) et termes "populaires » (zoku 俗) serait ainsi une tentative pour créer un haut vernaculaire japonais légitime, celui réalisé à haute voix dans les contextes rituels. On aurait ainsi un vernaculaire légitime, utilisable dans les récitations rituelles, mais qui ne serait pas détaché de la tradition académique des lettrés car il tire sa légitimité de l'équivalence avec les vocables sinitiques du dictionnaire ${ }^{7}$.

Arthur Defrance Doctorant à l'E.P.H.E., C.R.C.A.O.

1. Nous désignons par le terme «sinitique" ce que l'on appelle souvent le "chinois classique» ou le "chinois littéraire ", suivant Mair Victor, "Buddhism and the Rise of the Written Vernacular in East Asia: The Making of National Languages ", Journal of East Asian Studies, 53, n 3, 1994 : 707-751.
2. 1986 semble être une bonne année de départ, puisque c'est à la fois l'année de publication de la riche biographie de Sugawara no Michizane 菅原道真 par Robert Borgen (Sugawara no Michizane and the Early Heian Court, Honolulu, University of Hawai'i Press) et celle de l'essai de David Pollack (The Fracture of Meaning: Japan's Synthesis of China from the Eighth through the Eighteenth Centuries, Princeton, NJ, Princeton University Press). On retrouve dans ce corpus anglophone des auteurs comme John Wixted, David Lurie, Edward Kamens, Joshua Mostow, Jennifer Guest, Wiebke Denecke et Ivo Smits.

3. Voir son «Kanbun, Histories of Japanese Literature and Japanologists ", Sino-Japanese Studies, $10, \mathrm{n}^{\circ} 2,1998$ : 23-31.

4. Le texte a fait l'objet dès 1961 d'une traduction en allemand - langue que Steininger semble maîtriser - par Inge-Lore Kluge ("Die Denkschrift in drei Artikeln des Sugawara Fumitoki aus dem Jahre 958 ", Oriens Extremus, 8, $\mathrm{n}^{\circ} 2$, décembre 1961 : 223-232). Le texte est aussi discuté plus récemment par Antonio Manieri dans "Modelli di dissenso in Honchō monzui", in Migliore Maria Chiara et al., Il Dissenso in Giappone, Rome, Aracne : 75-112.

5. Whitman John, "The Ubiquity of Gloss ", SCRIPTA, 3, 2011 : 95-121.

6. Lurie David, Realms of Literacy. Early Japan and the History of Writing, Cambridge, Harvard University Asia Center, 2011.

7. Si nous sommes d'accord avec ce point, la comparaison avec le vernaculaire illustre, que Dante utilise pour son traité De l'éloquence vulgaire, paraît un peu discutable, dans la mesure où celui-ci ne tire pas sa légitimité d'un rapport avec le latin, mais du fait qu'il serait, comme le latin, une langue réglée (voir le chapitre viII de Mazzocco Angelo, Linguistic Theories in Dante and the Humanists, Leiden, Brill, 1993). 